\title{
Uncertainty and sensitivity analysis of flood risk management decisions based on stationary and nonstationary model choices
}

\author{
Balqis M. Rehan ${ }^{1,2, a}$, and Jim W. Hall ${ }^{1}$ \\ ${ }^{1}$ Environmental Change Institute, School of Geography and the Environment, University of Oxford, Oxford, United Kingdom \\ ${ }^{2}$ Civil Engineering Department, Faculty of Engineering, Universiti Putra Malaysia, 43400, Serdang, Malaysia
}

\begin{abstract}
Current practice in flood frequency analysis assumes that the stochastic properties of extreme floods follow that of stationary conditions. As human intervention and anthropogenic climate change influences in hydrometeorological variables are becoming evident in some places, there have been suggestions that nonstationary statistics would be better to represent the stochastic properties of the extreme floods. The probabilistic estimation of non-stationary models, however, is surrounded with uncertainty related to scarcity of observations and modelling complexities hence the difficulty to project the future condition. In the face of uncertain future and the subjectivity of model choices, this study attempts to demonstrate the practical implications of applying a nonstationary model and compares it with a stationary model in flood risk assessment. A fully integrated framework to simulate decision makers' behaviour in flood frequency analysis is thereby developed. The framework is applied to hypothetical flood risk management decisions and the outcomes are compared with those of known underlying future conditions. Uncertainty of the economic performance of the risk-based decisions is assessed through Monte Carlo simulations. Sensitivity of the results is also tested by varying the possible magnitude of future changes. The application provides quantitative and qualitative comparative results that satisfy a preliminary analysis of whether the nonstationary model complexity should be applied to improve the economic performance of decisions. Results obtained from the case study shows that the relative differences of competing models for all considered possible future changes are small, suggesting that stationary assumptions are preferred to a shift to nonstationary statistics for practical application of flood risk management. Nevertheless, nonstationary assumption should also be considered during a planning stage in addition to stationary assumption especially for areas where future change in extreme flows is plausible. Such comparative evaluations would be of valuable in flood risk management decision-making processes.
\end{abstract}

\section{Introduction}

In hydrology and water resources planning, stochastic methods are routinely applied in the design process. Conventionally, it is assumed that the extreme hydrological events are stationary, which means that the probability distribution of the extremes remains time invariant over the design life/appraisal period of the planned structure. The assumption is, however, argued due to the fact that the extremes hydrological time series such as extreme precipitation and extreme floods and drought are driven by a complex interaction of different factors that inevitably changes over time. For example, the hydrological cycle of river basins is affected by the changes in land use from human intervention and urbanization [1]. In some countries, changes in acceleration of runoff due to large-scale deforestation has causes unprecedented event of flooding.

There are also concerns over the effect of atmospheric circulation systems such as El Nino Southern Oscillation (ENSO), Pacific Decadal Oscillation (PDO) and North Atlantic Oscillation (NAO) to the pattern of observed extreme precipitation and extreme flood events [e.g. 2, 3]. Increased greenhouse gases in the atmosphere that result in climate change is another reason for concerns over changes in extreme hydrological variables that may effectively result in changes in frequency and magnitude of extreme floods and drought [4].

The significant impact of the mentioned factors to streamflow remains inconclusive [5]. Concerns over the possible nonstationarity and the impact of the conventional assumption of stationary in the case of designing flood protection are valid as the design structure are typically meant to be functional for decades. There has been a call to identify nonstationary probabilistic models instead of relying upon the stationary assumption in practical flood risk management problems [6]. Yet abandoning stationary probabilistic model and identifying approaches of nonstationary for practical use in flood risk management raises challenges to flood risk analysts and the research community $[7,8]$.

Concerns over nonstationarity have led many studies to conduct trend analysis on extreme precipitation and extreme streamflow observations [9]. A large number of

\footnotetext{
${ }^{\mathrm{a}}$ Corresponding author: balqis@upm.edu.my
} 
studies have also tried to address the changes in flood frequency by analysing changes over time slices of climate model-based future projections [e.g. 10, 11]. The findings from the analysis of change in future flows projections have been taken as the basis of design and planning guidelines for flood risk management in England and Wales [12]. Whereas many studies have relied on statistical trend tests such as Mann-Kendall trend test and regression analysis to observe change, there is a strong emphasis on the needs for developing more consistent non-stationary frequency analysis methods that can account transient nature of a changing climate [9].

The existence of nonstationarity in extreme streamflow is well recognized but research on the applicability of nonstationary model in flood frequency analysis is just recently being explored. A number of studies have conducted comparison analysis of design estimates from nonstationary probabilistic model to those of stationary [e.g. 13, 14, 15], which lead to emergence of diverse opinions. Some indicate preferences on nonstationary model with some cautions [13, 14, 16], whilst others discard the notion that stationary is dead and suggesting that stationary should still remain the default assumption [15].

In the face of uncertain future and the subjectivity of model choice, this study attempts to incorporate nonstationary probabilistic model in a flood risk management decision analysis framework, in addition to the conventional stationary model. Different sources of uncertainty is also addressed and well embedded in the simulation study. In demonstrating the practical implications of applying nonstationary model additional to stationary model, decision makers' behaviour in deciding upon an optimal protection level of flood protection is simulated. Decision uncertainty is represented by incorporating subjectivity of model choices between stationary and nonstationary probabilistic models in the decision making process. The work is implemented to a hypothetical case study where other components for cost-benefit analysis (i.e. cost and damage models) are derived accordingly to focus on the main aims of the study. The results are presented with explicit uncertainty range and the outcomes of the sensitivity analysis.

The paper is organised as follows. Section 2 presents the methodology for the study, which includes an integrated framework designed to represent decision makers' rationale approach in deciding upon an optimal protection. Results are presented in Section 3 whilst Section 4 presents the discussion and conclusion.

\section{Methodology}

A fully integrated framework of decision analysis was developed for the purpose of the study (Figure 1). The framework starts with identification of appropriate nonstationary underlying distribution to simulate long time series of extreme flow discharges (i.e. 150 years of annual maxima) (Figure 1 row 1). The simulated flow discharge is the basis of the exploration study. The first 50 years of the simulated flows is regarded as a 'historical' record and used as input in a decision making process via a risk-based optimisation methodology (RBOM). The methodology has been implemented in a number of case study and has been well-accepted in guiding for an optimal flood protection [e.g. 17, 18, 19]. Further explanation on RBOM is presented in Section 2.2 .

Conflicting scenarios of decision makers having different assumptions about the future underlying condition (i.e. stationary or nonstationary) are taken into consideration in the simulation study to allow comparison of outcomes. The differing option of either using a stationary or nonstationary model in the flood hazard characterisation is therefore embedded within the simulation framework (Figure 1 row 2). It is assumed that in the case of nonstationary model choice, if an upward rate of change is detected, then a nonstationary model is fitted in the RBOM, else stationary model would be used (Figure 1 row 3) reflecting decision makers' risk averse attitude.

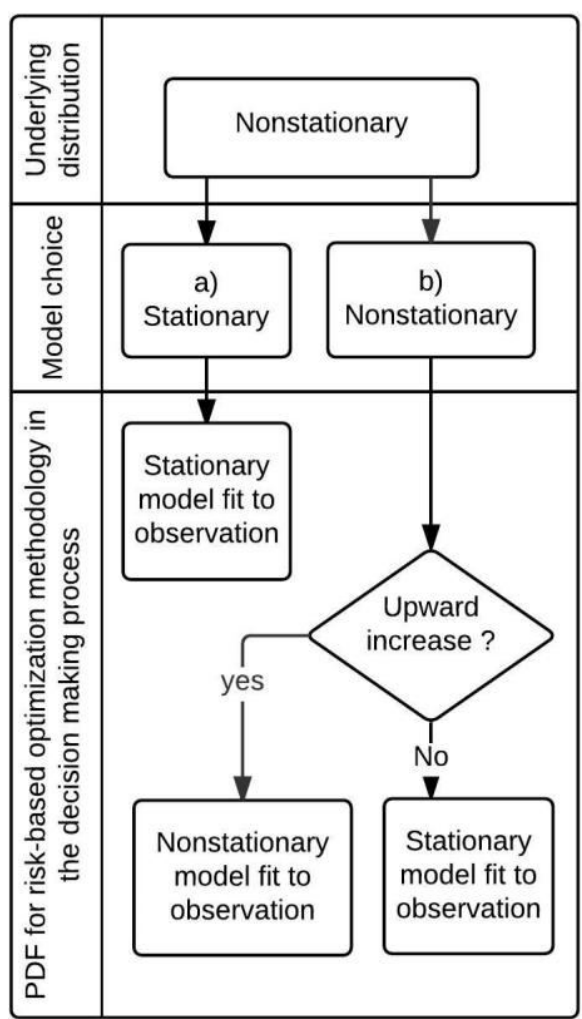

Figure 1: Work flow showing the state of the underlying distribution, the model choice by decision makers and the final model used in the risk-based optimization methodology

The performance of decisions made according to the model choice are inspected by comparing the simulated decisions (in design discharge unit) with the annual extreme flows simulated over a specified appraisal period, in this case referring to the final 100 years annual maxima. The uncertainty range of the decisions performance due to the natural variability and decision uncertainty is captured by repeating the experiment for multiple simulations through Monte Carlo simulation methodology. Furthermore, a range of different rate of change is considered to examine the sensitivity of the decisions performance to the different plausible future. In 
addition, the economic performance of decisions based on partial information is compared with that of perfect information

Section 2.1 presents the characteristics of the nonstationary underlying distribution used to simulate the extreme flow series. In Section 2.2, the risk-based decision making process used to simulate decision makers behaviour is briefly introduced. The economic indicators used to evaluate the performance of decisions are presented in Section 2.3.

\subsection{Nonstationary underlying distribution and simulation of annual maxima flows}

In this study, a nonstationary Generalized Extreme Value (GEV) model with time as covariate was chosen to simulate the annual maxima flow series. Assuming that the location parameter is linearly changing over time, the inverse cumulative distribution function (CDF) of the nonstationary GEV distribution was used to generate the flows. The CDF according to Jenkinson [20] can be written as follows:

$$
F_{X}(x)=\exp \left\{-\left[1-\kappa\left(x-u_{t}\right) / \alpha\right]^{1 / \kappa}\right\}
$$

where,

$$
u_{t}=u_{o}+u_{l}(t)
$$

$u_{t}$ is the location parameter with time as covariate, $\alpha$ is the scale parameter and $\kappa$ is the shape parameter. The location parameter as a linear trend has an initiation of $u_{\mathrm{o}}$ and a rate of change $u_{l}$ over $t$.

The generation of annual maxima flow series from the function should use realistic parameter values. For this study, available historical records of the Thames at Kingston gauging station ranging from 1883 to 2012 were fit into the model using L-moments [21]. Initial check using the Akaike Information Criterion reveals that the time series is best represented by a GEV stationary model (i.e. without covariate) whilst the second best model is the GEV nonstationary model with a linear location parameter outperforming GEV nonstationary model with log-scale parameter. As the aim of the study is to evaluate the effects of nonstationary underlying distribution, estimated parameters of the nonstationary GEV distribution with linear location parameter were used to simulate the annual maxima flow series. The associated parameters estimated by maximum likelihood estimators are $u_{o}=250.65, u_{l}=0.2, a=95.7, \kappa=-0.046$

11 different scenarios of future change were considered to test the sensitivity of outcomes. This is undertaken by specifying different changes of discharges per year $\left(u_{1}\right)$ over the appraisal period for each scenario, ranging from 0 to $1.0 \mathrm{~m}^{3} / \mathrm{s}$ increase of discharge annually. The scenarios therefore is set to have a different rate of change by a factor of $0.1 \mathrm{~m}^{3} / \mathrm{s}$, which means that the simulated future may have a weaker or greater representation of change over the future period as compared to the simulated historical underlying distribution $\left(u_{1}=0.2 \mathrm{~m}^{3} / \mathrm{s} /\right.$ year $)$. Hence future underlying distribution with $u_{1}=0.2 \mathrm{~m}^{3} / \mathrm{s} /$ year has the same rate of change as the simulated historical underlying distribution. Note that $1.0 \mathrm{~m}^{3} / \mathrm{s}$ increase per year will cause a $100 \mathrm{~m}^{3} / \mathrm{s}$ increase of discharge at the end of the 100 years appraisal period. This is extremely high and a higher value might not worth to be considered.

In order to simulate smooth transaction of values over the 'historical' and 'future' period, the location parameter, $u$, of the 'future' underlying distribution at the transition time $(t=51)$ should be the same as that of 'historical' underlying distribution. However due to the different rate of change between the two periods, $u_{o}$ of those periods are unique. Table 1 shows the computed $u_{o}$ associated with an assigned $u_{1}$ for the future period. To have an insight on the relative variability of the simulated annual maxima from different $\mathrm{u}_{1}$, tabulations of average annual maxima flows across 150 years when the historical $u_{1}=0.2 \mathrm{~m}^{3} / \mathrm{s} /$ year and the future $u_{1}$ is 0.4 and $0.7 \mathrm{~m}^{3} / \mathrm{s} /$ year respectively are given in Figure 2 .

\begin{tabular}{|l|l|l|l|l|l|l|}
\hline $\begin{array}{l}\boldsymbol{u}_{\mathrm{t}=51} \\
\left(\mathrm{~m}^{3} / \mathrm{s} / \mathrm{year}\right)\end{array}$ & $\mathbf{0}$ & $\mathbf{0 . 1}$ & $\mathbf{0 . 2}$ & $\mathbf{0 . 3}$ & $\mathbf{0 . 4}$ & $\mathbf{0 . 5}$ \\
\hline
\end{tabular}

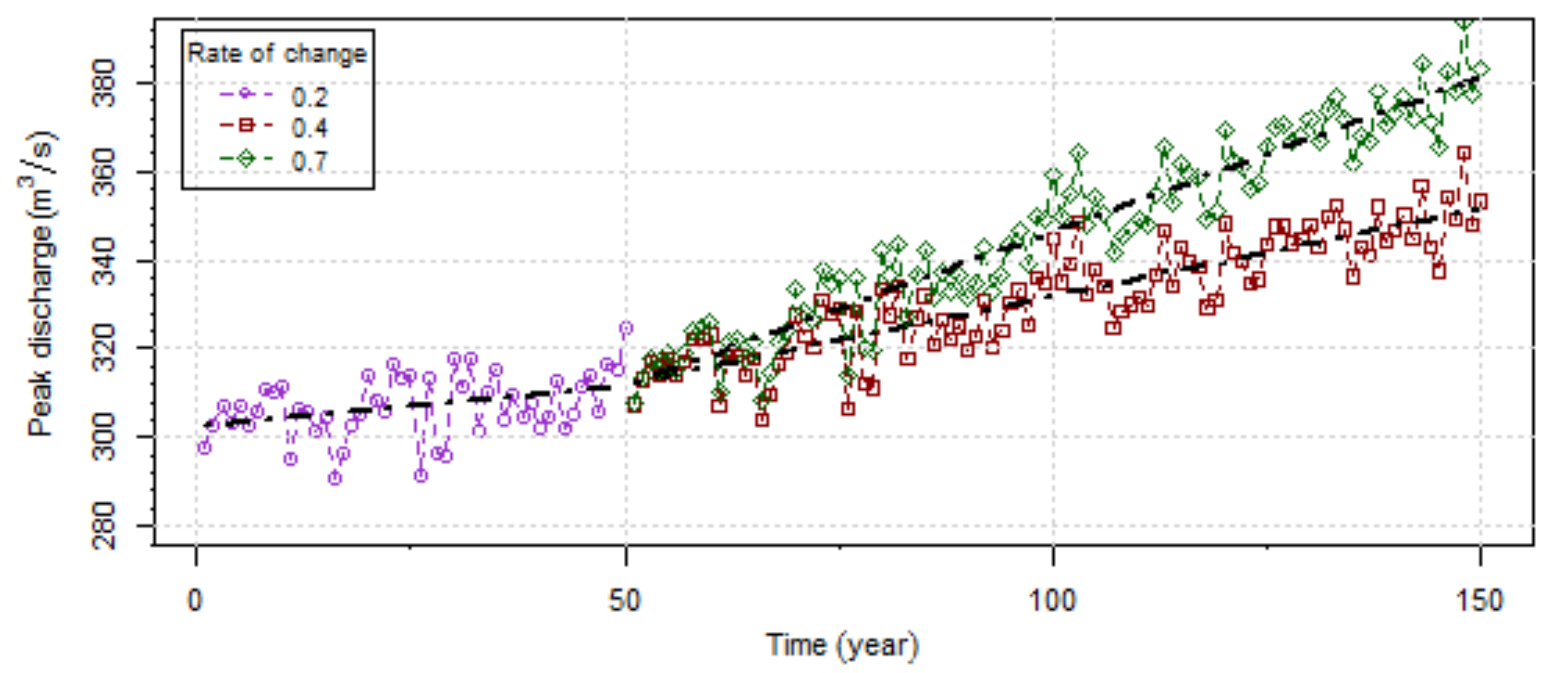

Figure 2: Mean annual maxima flows associated with time over historical and future period for 300 simulated flow series. 


\begin{tabular}{|l|l|l|l|l|l|l|}
\hline $\boldsymbol{u}_{0}\left(\mathbf{m}^{\mathbf{3}} / \mathbf{s}\right)$ & $\begin{array}{l}260 . \\
85\end{array}$ & $\begin{array}{l}255 . \\
75\end{array}$ & $\begin{array}{l}250 . \\
65\end{array}$ & $\begin{array}{l}245 . \\
55\end{array}$ & $\begin{array}{l}240 . \\
45\end{array}$ & $\begin{array}{l}235 . \\
35\end{array}$ \\
\hline $\begin{array}{l}\boldsymbol{u}_{\mathrm{t}=\mathbf{5 1}} \\
\left(\mathbf{m}^{\mathbf{3}} / \mathbf{s} / \mathbf{y e a r}\right)\end{array}$ & $\mathbf{0 . 6}$ & $\mathbf{0 . 7}$ & $\mathbf{0 . 8}$ & $\mathbf{0 . 9}$ & $\mathbf{1 . 0}$ & \\
\hline $\boldsymbol{u}_{0}\left(\mathbf{m}^{\mathbf{3}} / \mathbf{s}\right)$ & $\begin{array}{l}230 . \\
25\end{array}$ & $\begin{array}{l}225 . \\
15\end{array}$ & $\begin{array}{l}220 . \\
05\end{array}$ & $\begin{array}{l}214 . \\
95\end{array}$ & $\begin{array}{l}209 . \\
85\end{array}$
\end{tabular}

Table 1: Summary of the nonstationary GEV location parameter components for the underlying future 'design life' associated to the rate of change at $t=51$.

\subsection{Risk-based decision making process}

Upon simulating the decision making process, the study has adopted the well-known risk-based optimization methodology (RBOM). One of the key activities in implementing the methodology is the estimation of residual risk over the design life/appraisal period of the proposed project in economic term.

Risk can be defined as the product of extreme flows probability and the consequent damage, whereby the probability distribution function (PDF) is integrated together with an appropriate damage function [22]. The risk estimation following this definition provides the advantage of integrating both the hazard characteristic and the economic damage into the evaluation, hence complementing for a cost-benefit analysis that satisfies a more practical approach in flood risk decision-making. Furthermore, the adoption of the risk-based concept allows a fair comparison of outcomes between stationary and nonstationary model choices because the risk is computed based on interval estimates rather than point estimates.

The PDF is identified through the flood frequency estimation and available historical extreme flow dataset is used as input. For this study, it is assumed that the decision makers adopt the GEV distribution and maximum likelihood to estimate the distribution parameters. For the case of preferred stationary model, the PDF of the GEV distribution $f$ (.) with notations similar to that of Equation 1 can be denoted as [20]:

where,

$$
f(x \mid u, \alpha, \kappa)=\frac{1}{\alpha} y^{1-\kappa_{e}-y}
$$

$$
y= \begin{cases}\left(1-\frac{\kappa(x-u)}{\alpha}\right)^{\frac{1}{k}}, & \kappa \neq 0 \\ e^{-\frac{x-u}{\alpha},} & \kappa=0\end{cases}
$$

For the cases where nonstationary model choice is preferred, the location parameter would be changing over time, hence $u$ follows that of $u_{t}$ as given in Equation 2 . The likelihood function with parameters $\boldsymbol{\theta}$ can be denoted as follows [23] :

$$
\ell(X ; \boldsymbol{\theta})=\prod_{i=1}^{m} f_{X}\left(x_{i} \mid \boldsymbol{\theta}\right)
$$

where $m$ is the sample size compiling observations of $x_{1}$, $x_{2}, \ldots, x_{m}$. To estimate the parameters, log-likelihood of the function is maximized with respect to parameter vector by numerical solutions. The statistical model fitting is programmed using an inbuilt 'extRemes' toolkit [24] in R software.
A simplified damage function in flood impact assessment often represented as either linear, quadratic or square root function [25]. This study takes the damage function as a square root function. The damage functions are separated into three scenarios. No damage scenario ( $q$ $\leq q_{o}$ or $q \leq \hat{q}$ ), damaging flood scenario conditioned to allocated protection design $(q>\hat{q})$ and damaging flood scenario conditioned to without project $\left(q>q_{o}\right)$, where $\hat{q}$ is the protection design and $q_{o}$ is the status quo discharge level of no damage if flow discharge is equal or below the level. Constant $a$ and a stage-discharge function $h_{d}($. were used to obtain realistic values of flood damages.

$$
D_{t}(q)=\left\{\begin{array}{c}
0, \text { for }_{d}=0, q \leq q_{o} \text { or } q \leq \hat{q} \\
a\left(h_{d}(q)\right)_{t}^{0.5}, \text { for } h_{d} \neq 0, q>\hat{q} \\
a\left(h_{d}(q)\right)_{t}^{0.5}, \text { for }_{d} \neq 0, q>q_{0}
\end{array}\right.
$$

The function has been derived according to a hypothetical case study in Balqis and Hall [26] and is adopted for this study.

Combining the PDF and damage function, the risk function $R_{t}$ in annual economic term can be estimated by solving

The

$$
R_{t}=\int_{\hat{q}}^{q_{L}} \rho_{t}(q) D_{t}(q) d q \quad \text { subscript }
$$

represents the possible changes in time, in this case in yearly time scale. The lower and upper limit of integration is in the unit of flow discharge (i.e. $\mathrm{m}^{3} / \mathrm{s}$ ). It is important to note that the integration is to be numerically solved, hence an infinite upper limit if exists from an unbounded PDF will be numerically mapped onto an appropriate finite interval with infinity as the limit.

The decision-making process through the RBOM follows a framework of cost-benefit analysis. However, instead of having the optimal protection by identifying the minimum net present value (NPV), the RBOM identifies the minimum of total present value cost (TPVC) for the optimal protection. Through the RBOM, datasets of investment cost and residual risk posed by a range of possible protection design are incorporated into the assessment for identification of the minimum TPVC.

The project cost function $C_{t}$ is taken as a power function given by

$$
C_{t}(q)=\left\{\begin{array}{lr}
0, & \text { for }_{c}-h_{g}=0 \\
A\left(h_{c}(\hat{q})-h_{g}\left(q_{0}\right)\right)^{\bar{B}}, \text { for }_{c}-h_{g} \neq 0
\end{array}\right.
$$

The cost is determined by the height of the flood defence crest level from the river base $\left(h_{c}\right)$ and the status quo threshold level $\left(h_{g}\right)$, which is triggered by $\hat{q}$ and $q_{o}$ respectively. To have realistic values of investment costs, $A$ and $B$ constants are considered. Similar to the damage function, the cost function that has been derived in Balqis and Hall [26] is adopted for this study.

By solving the residual risk function and the cost function for each of the protection design considered, the TPVC can be calculated by discounting the total costs over the appraisal period. The optimal protection design is ultimately the minimum value of the TPVC of the 
considered range of protection design that can be denoted as an objective function of an optimization procedure as follows:

$$
\min _{\bar{q}} T P V C=\sum_{t=0}^{n_{s t r}} \frac{1}{(1+r)^{t}}\left(R_{t}(\hat{q})+C_{t}(\hat{q})\right)
$$

where $r$ is the discount rate and $n_{s t r}$ is the total appraisal years. The discount rate is taken as $3.5 \%$ following the standard rate as suggested in the treasury [27].

The cost and damage functions associated with the flood protection measures and the flood risk system should be derived based on different types of contributing costs and spatial distribution of elements at risk. However, the present study focuses on the sensitivity of the results, hence the absolute values of the investment cost and flood damage will be arbitrary from the representative functions.

\subsection{Economic evaluation of decisions performance}

The effects of the stationary and nonstationary model choices to the decisions' economic performance were evaluated in two ways; (1) through Monte Carlo simulation methodology in capturing the uncertainty range of outcomes, and (2) through the use of perfect information about the simulated future to obtain the expected value of the decisions performance. This is to allow comparisons between the results from the Monte Carlo simulation and the results from the first-order analysis.

Section 2.1.1 presents the way the economic performance is quantified using the generated flows over the appraisal period, whilst Section 2.1.2 presents the way the economic performance of decisions are quantified using the PDF of the underlying distribution of the appraisal period.

\subsubsection{Quantification based on annual maxima simulation}

After the decision-making process has been simulated, it is assumed that the estimated optimal protection is followed and constructed accordingly. The net present value (NPV) is adopted in testing the economic performance of the protection design associated with decision makers' model choice, the underlying 'historical' distribution and the underlying 'future' distribution. The two important components for the NPV quantification are the streams of benefit and the investment costs of the project as denoted below

$$
N P V=\sum_{t=0}^{n_{s t r}} \frac{1}{(1+r)^{t}}\left[\left(B_{t}(q)\right)-C_{t}(\hat{\mathrm{q}})\right]
$$

where,

$$
B_{t}(q)=D_{t}(q>\hat{q})-D_{t}\left(q>q_{0}\right)
$$

The benefit associated with the decision makers' previous decision is quantified using pair-wise comparison of each of the simulated 'future' annual maxima flows (q) of 100 years appraisal period with the protection threshold. For scenario of with-protection, flows that exceed the protection design will contribute to flood damage for that particular year, whilst for scenario of do-nothing, flood damage would be calculated when the simulated flows exceeding the existing flood damage threshold $q_{o}$. The benefit of having the protection design is then calculated by taking the difference between the damage of with-project and the damage of do-nothing condition.

Note that the way the NPV is computed as previously mentioned takes into account the annual maxima flow series simulated for the future. Because the study implements multiple simulations from the same underlying distribution, a range of possible NPV will be obtained that allows for the uncertainty range to be tabulated and observed.

\subsubsection{Quantification based on PDF of the underlying distribution}

In computing the expected economic performance of the decisions without uncertainty range, the NPV equation is still denoted as equation 6 , but the way the benefit of having the protection measures is computed for the NPV uses the exact form of the PDF. The benefit thus can be denoted as:

$$
B_{t}(q)=R_{t_{T_{r} q_{o}}}(q)-R_{t_{T, q}}(q)
$$

where $T$ represents the underlying characteristic of the 'future' distribution function, hence is unique for every scenarios considered.

\section{Results}

The nonstationary underlying distribution that has been parameterized based on the long time series of Thames at Kingston historical records and the assigned rate of change (Section 2.1) were used to generate 300 sets of 'historical' and 'future' annual maxima flow series. The number of simulations was arbitrary chosen. Altogether, 11 scenarios were considered all with fixed rate of change for the historical underlying distribution $\left(u_{l}=0.2 \mathrm{~m}^{3} / \mathrm{s}\right)$, but each scenario- with varying rate of change for the future underlying distribution.

As a first instance, the risk-based optimization methodology with stationary (SE) and nonstationary (NSE) model choice was implemented for the 300 sets of the simulated 'historical' records. The optimal protection design based on the model choice and the corresponding investment costs were estimated using Equation 8 and the results were tabulated in histograms and box-plots as shown in Figure 3 and 4. The red dotted vertical line overlaying the histograms represents the optimal design (i.e. $456 \mathrm{~m}^{3} / \mathrm{s}$ ) based on the perfect information of the historical underlying distribution (POP). 


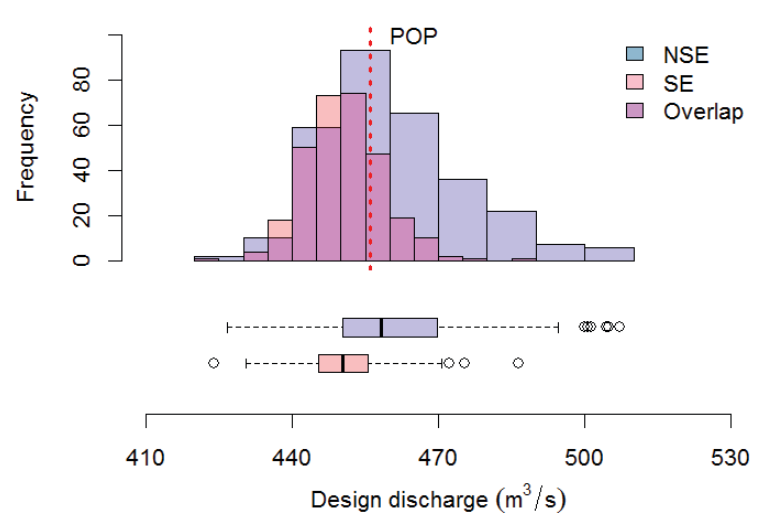

Figure 3: histograms and boxplots of optimal protection design estimated based on nonstationary (NSE) and stationary (SE) model choices. The red line refers to optimal protection design based on perfect information (POP).

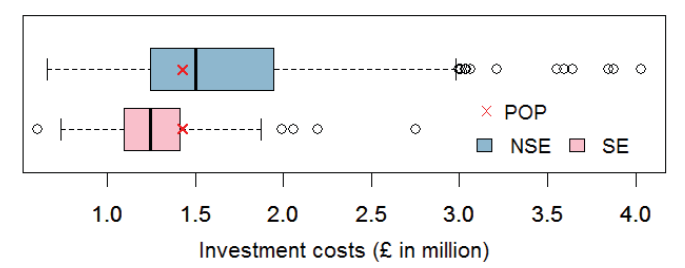

Figure 4: boxplots of investment costs of decisions based on nonstationary (NSE) and stationary (SE) model choices. The red crosses refer to investment costs based on perfect information (POP).

In the probability theory, the expected value from the long-run average value should converge to the value of POP. However, as can be seen from Figure 3 and 4 the median values from the NSE model do not converge to the values from the POP. In fact, the distribution from NSE does not converge to asymptotic normal distribution. Although a higher number of simulations might improve the result, a subsequent test using 500 simulations improve the bias of the expected value by only $0.09 \%$ from that of 300 simulations, which can be considered insignificant. It is therefore decided that the experiment will be preceded with the initial 300 simulation sets.

Despite the existing bias from the sampling error regardless the model choice, a smaller bias is obtained from NSE as compared to SE. The smaller bias from NSE is consistent with what may be expected from applying the same model representation in the decisionmaking as to that of future scenario (i.e. $0.2 \mathrm{~m}^{3} / \mathrm{s} /$ year). However, the variance in the outcomes is higher for the NSE as compared to SE. This behaviour is expected as the nonstationary model has a higher number of parameters as compared to SE [28].

The study is continued by working with the simulated datasets of the 11 considered scenarios of future change. As to have an insight on the possible range of flood damage upon do-nothing condition, the simulated 'future' extreme flows were fed into the damage function with the do-nothing flow threshold as the point of reference. Figure 5 shows the range of total flood damage in economic terms that can be expected from each of the considered future scenarios of change. Higher median values of flood damage can be seen from the future that has a higher rate of change of extreme flow discharge.

The risk-based optimization methodology with SE and NSE model choice respectively is implemented independently to each of the 300 historical realization sets of the 11 future change scenarios. Flood damage reduction of having the optimal protection associated with the SE or NSE model choice and the future underlying distribution was then quantified by solving Equation 10. Subsequently, the NPV for each simulation set was computed using Equation 9.

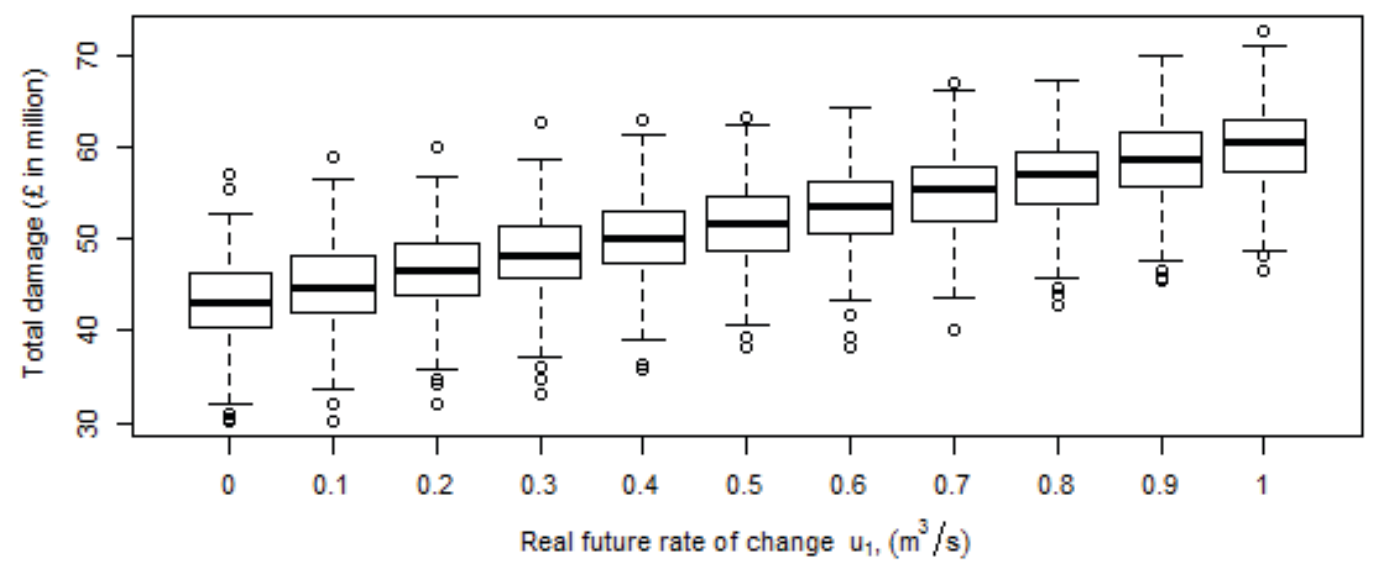

Figure 5: Distributions of total damage of do-nothing over the design life of protection, associated with nonstationary and stationary estimates respectively for different possible future $\boldsymbol{u}_{1}$.

The resulting total benefit over the appraisal period for each simulation set corresponded to each future scenario were recorded and tabulated accordingly as presented in Figure 7. The outcomes of NPV were similarly tabulated according to the considered scenarios as illustrated in Figure 8. To compare the results of NPV based on realization values and those of the POP, the NPV based on the POP were solved using Equation 9 
based on risk reduction benefit of Equation 11. The results were tabulated in the same figure as to those from simulations to allow direct comparison (Figure 8).

From Figure 6, the total damage caused by SE model choice when in fact the future is nonstationary exhibits higher median and maximum values as compared to the total damage obtained from the NSE model choice. The behaviour is most likely due to the condition of under protection entailed the SE model choice. In return, this has causes lower total benefits for SE-based protection design as compared to those of NSE for all scenarios. Nevertheless, the median of total damage and total benefit regardless the model choice show upward trends across higher rate of change, suggesting the higher benefit of protection against flood for much intensifying future change despite the model error.

The median of NPV in Figure 8 shows similar behaviour with higher values for higher 'future' rate of change. The results reflect the effects of higher total benefits for higher rate of change and fix investment costs with respect to the SE and NSE model choice in the decision making process across all future scenarios considered. In addition, the positive NPVs from all possible decisions under all considered scenarios indicate the robustness of the risk-based optimization intervention approach used in the decision making process.

It is intriguing to find that using a stationary model in the decision making process for unprecedented nonstationary future result in only slightly less expected total benefits of risk reduction and NPV as compared to the results when the nonstationary model is used. All scenarios of change that have been considered in this study including the most extreme case of change $\left(u_{l}=1\right.$ $\mathrm{m}^{3} / \mathrm{s} /$ year) show that the discrepancies between the model choices are small. The behaviour is likely caused by fluctuations of stationary distribution near the nonstationary one that consequently makes the results close to each other. Similar minor effects between the stationary and nonstationary models have been mentioned and demonstrated in [15] using a range of return periods and associated bootsrap 95\% confidence interval.

Further comparison between the expected values of NPV from perfect information with the ones from estimated distribution parameters (Figure 8) indicates that the results from the estimated distribution parameters are converging to the ones from parent distribution, increasing the confidence in the simulation setup and the resulting outcomes.

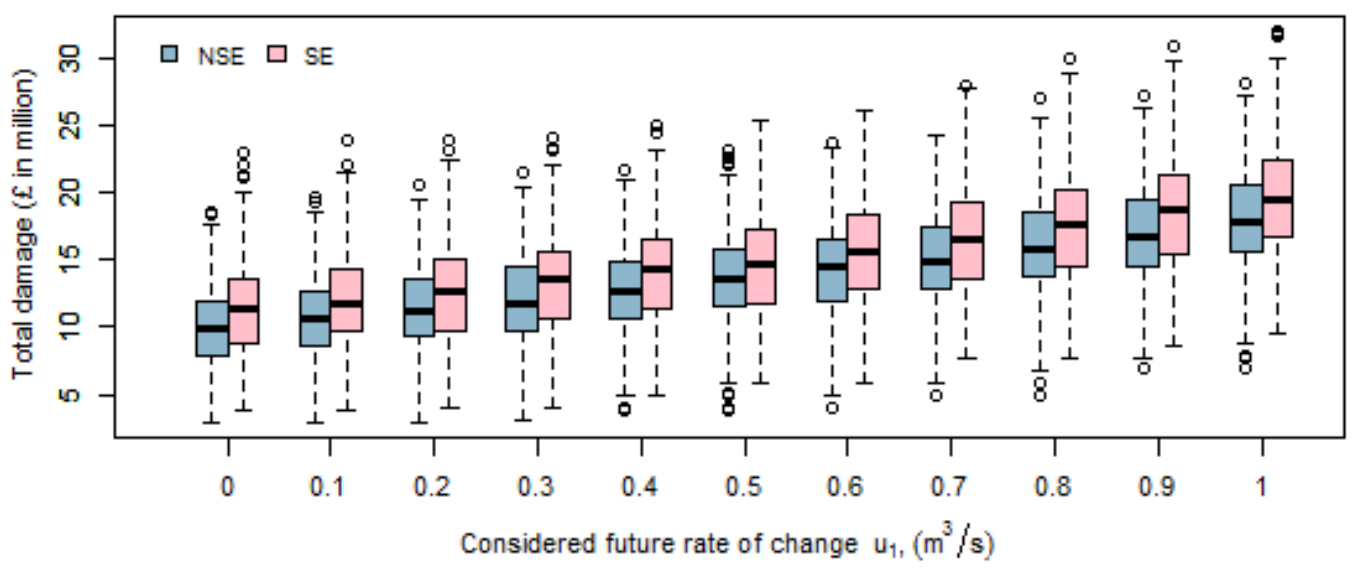

Figure 6: Distributions of total damage of with-project over the design life of protection, based on nonstationary and stationary estimates respectively over different possible future $u$.

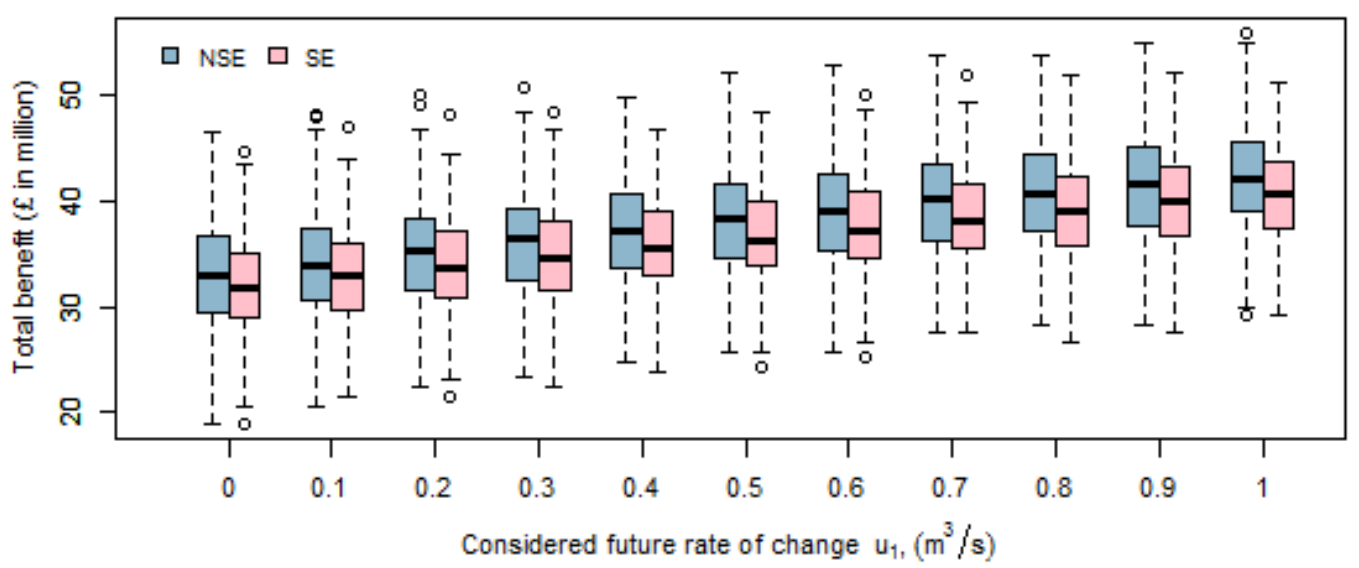

Figure 7: Distributions of total benefit of with-project over the design life of protection, based on nonstationary and stationary estimates respectively for different possible future $u_{l}$. 


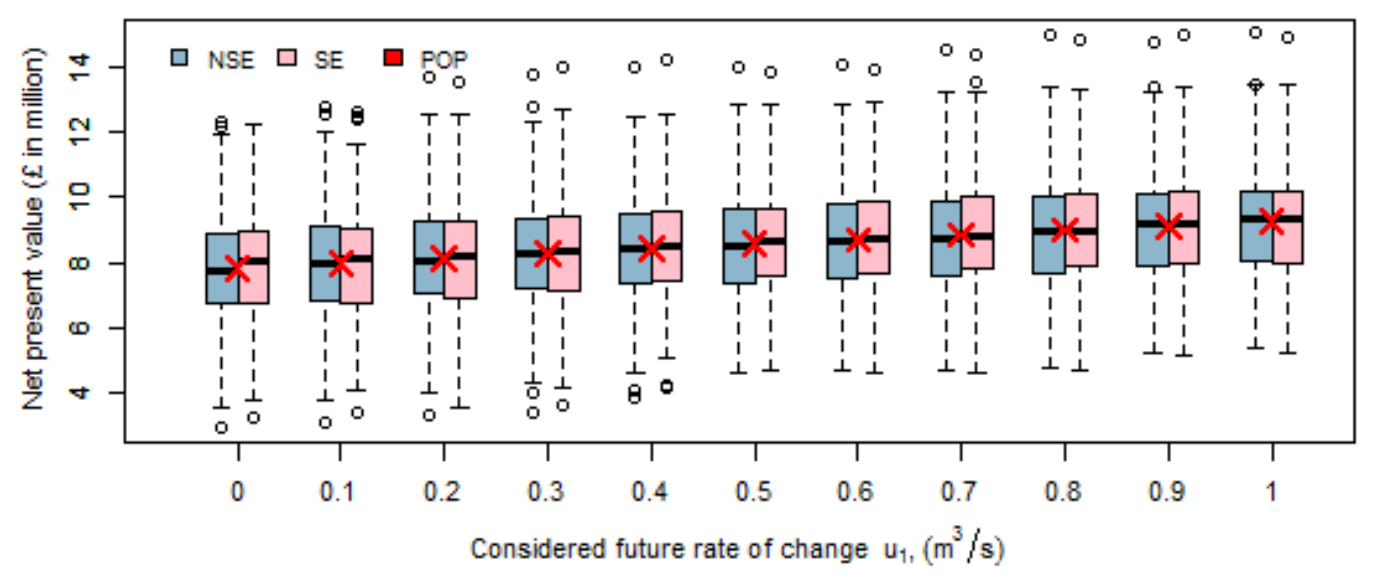

Figure 8: Distributions of NPV of with-project over the design life of protection, based on nonstationary and stationary estimates respectively for different possible future $u_{l}$

\section{Discussion and conclusion}

The paper has demonstrated the effects of stationary and nonstationary model choices against a number of nonstationary future scenarios in terms of their economic performances. Risk-based optimization methodology has been adopted in the decision making process. The results reveal several profound findings that are highlighted in the subsequent paragraphs.

The higher variance in the estimated optimal protection from nonstationary model choice that result in a higher uncertainty range as compared to stationary model choice may give preference to the stationary model in flood frequency analysis despite the possible nonstationary future change. Furthermore, the use of a stationary model as compared to a nonstationary model in estimating the optimal decision for future that is unknowingly exhibit nonstationary only brings to minor discrepancies in the total benefit and NPV. The results suggest that stationary model remains relevant in preparing for future change despite the potential model error. This further echoes a strong suggestion in [15] confirming that stationarity should remain the default assumption even when the evidence for nonstationarity is apparent.

However, it should be stressed here that the preference on stationary models over nonstationary models does not mean that nonstationary models should be discarded [16]. If evidence/prediction of future change in extreme flows is apparent and modelling capabilities exist, a decision analysis incorporating nonstationary models in addition to stationary models should be undertaken to have prior insights on the relative outcomes corresponding to the models used. Such additional information would be of valuable in the decision-making under uncertainty of flood risk management.

For practical application in decision making of longterm flood protection, the study reveals that the riskbased approach is promising in ensuring robust decisions in the face of uncertain future change. Unlike the conventional fix target protection (e.g. return period), the risk-based approach considers a wide range of possible flood events and their possible consequences, whilst evaluating a range of flood protection levels. The approach allows a cost-effective solution to be estimated through the optimization procedure within the RBOM.

The Monte Carlo simulation methodology for uncertainty range quantification allows the influence of natural variability additional to the model error to be explicitly addressed. However, it is influenced by the parameters of the parent distribution. This study has adopted the available long time series of Thames at Kingston historical records for a reliable parameterization of the PDF. Smaller sample size would inevitably cause discrepancies. However, reliable outcomes can be obtained when using sample size of 50 years as demonstrated in this study. Similar exploration, therefore, can be undertaken for other cases with relatively small sample size.

Currently, the study has been expanded to include climate model based projections of future flows for the case study area to compare the application of different information sources, i.e. directly from historical hydrometric records and from the climate model projections, and the effects of the distinct information to the decisions performance. Initial statistical tests undertaken for the climate model based projections future flows have revealed that there is a possibility of a preferred nonstationary model when using the climate model based projections of future flows. Hence, exploration of decisions performance involving the nonstationary statistics based on climate model projections additional to the conventional stationary assumption would provide valuable insights.

Since the outcomes of the economic performances depend on the behaviour of the probability distributions used in the decision-making, it is necessary to investigate the discrepancies and patterns of fluctuation in the probability distribution of stationary and nonstationary condition. The effects of the representation of the probability distributions to the ultimate economic performance can thus be investigated more rigorously and brings to a better understanding. 


\section{References}

1. Kundzewicz, Z.W., Hirabayashi, Y. and Kanae, S. (2010) River Floods in the Changing ClimateObservations and Projections. Water Resources Management, 24 (11), pp. 2633-2646

2. Khaliq, M.N., Ouarda, T.B.M.J., Ondo, J.C., Gachon, P. and Bobee, B. (2006) Frequency analysis of a sequence of dependent and/or non-stationary hydro-meteorological observations: A review. Journal of Hydrology, 329 (3-4), pp. 534-552

3. Chen, Z. and Grasby, S.E. (2009) Impact of decadal and century-scale oscillations on hydroclimate trend analyses. Journal of Hydrology, 365 (1-2), pp. 122133

4. IPCC (2012) Managing the Risks of Extreme Events and Disasters to Advance Climate Change Adaptation. A Special Report of Working Groups I and II of the Intergovernmental Panel on Climate Change [Field, C.B., V. Barros, T.F. Stocker, D. Qin, D.J. Dokken, K.L. Ebi, M.D. Mastrandrea, K.J. Mach, G.-K. Plattner, S.K. Allen, M. Tignor, and P.M. Midgley (eds.)]. Cambridge, UK, and New York, NY, USA: Cambridge University Press.

5. Kundzewicz, Z.W., Kanae, S., Seneviratne, S.I., Handmer, J., Nicholls, N., Peduzzi, P., Mechler, R., Bouwer, L.M., Arnell, N., Mach, K., Muir-Wood, R., Brakenridge, G.R., Kron, W., Benito, G., Honda, Y., Takahashi, K. and Sherstyukov, B. (2013) Flood risk and climate change: global and regional perspectives. Hydrological Sciences Journal, 59 (1), pp. 1-28

6. Milly, P.C.D., Betancourt, J., Falkenmark, M., Hirsch, R.M., Kundzewicz, Z.W., Lettenmaier, D.P. and Stouffer, R.J. (2008) Climate change: Stationarity is dead: Whither water management? Science, 319 (5863), pp. 573-574.

7. Galloway G. E. (2011). If stationarity is dead, what do we do now? Journal of the American Water Resources Association, 47(3), 563-570

8. Merz S. B., Hall J., Disse M. and Schumann A. (2010). Fluvial flood risk management in a changing world. Natural Hazards and Earth System Sciences, 10(3), 509

9. Madsen H., Lawrence D., Lang M., Martinkova M. and Kjeldsen T. R. (2013). A review of applied methods in Europe for flood frequency analysis in a changing environment. Prepared for WG4: Flood frequency estimation methods and environmental change for European cooperation in science and technology (COST). http://nora.nerc.ac.uk/501751/ [Accessed 2015]

10. Bell, V.A., Kay, A.L., Cole, S.J., Jones, R.G., Moore, R.J. and Reynard, N.S. (2012) How might climate change affect river flows across the Thames Basin? An area-wide analysis using the UKCP09 Regional Climate Model ensemble. Journal of Hydrology, 442, pp. 89-104.

11. Kay, A.L. and Jones, D.A. (2012) Transient changes in flood frequency and timing in Britain under potential projections of climate change. International Journal of Climatology, 32 (4).

12. Great Britain, Environment Agency (2016). Adapting to climate change: guidance for risk management authorities. [Online]. Available from: https://www.gov.uk/government/uploads/system/uplo ads/attachment_data/file/504245/LIT_5707.pdf [Accessed 7 March 2016]

13. Seidou O., Ramsay A. and Nistor I. (2012), Climate change impacts on extreme floods II: Improving flood future peaks simulation using non-stationary frequency analysis. Natural Hazards, 60(2), 715-726

14. Salas J. D. and Obeysekera J. (2014). Revisiting the concepts of return period and risk for nonstationary hydrologic extreme events. Journal of Hydrologic Engineering, 19(3), 554-568

15. Serinaldi F. and Kilsby C. G. (2015). Stationarity is undead: Uncertainty dominates the distribution of extremes. Advances in Water Resources, 77, 17-36

16. Milly P. C. D., Betancourt J., Falkenmark M., Hirsch R. M., Kundzewicz Z. W., Lettenmaier D. P., Stouffer R. J., Dettinger M. D. and Krysanova V. (2015). On critiques of 'Stationarity is dead: Whither water management?' Water Resources Research, 51(9), 7785-7789

17. Tung Y. K. (2005). Flood defense systems design by risk-based approaches. Water International, 30(1), 50-57

18. Jonkman S. N., Kok M., Van Ledden M. and Vrijling J. K. (2009). Risk-based design of flood defence systems: A preliminary analysis of the optimal protection level for the New Orleans metropolitan area. Journal of Flood Risk Management, 2(3), 170181

19. Rosner A., Vogel R. M. and Krishen P. H. (2014). A risk-based approach to flood management decisions in a nonstationary world. Water Resources Research, 50(3), 1928-1942

20. El Adlouni S., Bobee B. and Ourda T. B. M. J. (2008). On the tails of extreme event distributions in hydrology. Journal of Hydrology, 355(1-4), 16-33

21. Hosking J. R. M. (1997). Regional flood frequency analysis: An approach based on L-moments, Cambridge University Press.

22. Hall J. W. (2014). Flood risk management decision making under uncertainty. Applied Uncertainty Analysis for flood risk management, Imperial College Press, England, London, pp. 3-24

23. Coles S. (2002). An introduction to statistical modeling of extreme values, Springer, London.

24. Gilleland E. and Katz R. W. (2011). New software to analyze how extremes change over time. Eos. Transactions American Geophysical Union, 92(2), $13-14$

25. Boettle M. (2011). About the influence of elevation model quality and small-scale damage functions on flood damage estimation. Natural Hazards and Earth System Sciences, 11(12), 3327-3334

26. Balqis M. R. and Hall J. W. (2014). Flood risk management decision analysis with finite historical records and highly variable climate effects. Vulnerabilty, uncertainty and risk quantification, mitigation and management, Liverpool, UK, pp. 2267-2879

27. Great Britain, HM Treasury (2011). The Green Book: Appraisal and evaluation in Central Government. Treasury Guidance. [Online] 
https://www.gov.uk/government/uploads/system/upl oads/attachment_data/file/220541/green_book_comp lete.pdf [Accessed 7/3/2015]
28. Di Baldassarre G., Laio F. and Montanari A. (2009). Design flood estimation using model selection criteria. Physics and Chemistry of the Earth, Parts $\mathrm{A} / \mathrm{B} / \mathrm{C}, \mathbf{3 4}(\mathbf{1 0 - 1 2})$, 606-611 